\title{
Especulación inmobiliaria e identidad agrícola en una comunidad de ascendencia maya
}

Real estate speculation and agricultural identity in a community of Mayan descent

Especulação imobiliária e identidade agrícola em uma comunidade de ascendência maia

\author{
Itzel Rubi Díaz Tinoco \\ diaz.tinoco333@gmail.com \\ Estudiante de Doctorado CIESAS Peninsular - México \\ https://orcid.org/0000-0002-3232-6385
}

“...y así de rápido, no tardó, la tierra volvió a manos del patrón $"$

\begin{abstract}
RESUMEN
Hablar del ejido en México nos remite a un cúmulo de ideas asociadas a elementos re y post revolucionarios, relacionados en gran medida a la visibilización de actores sociales con poca, o nula participación económica, carente de posibilidades de desarrollo social, así como de maniobras neoliberales de reconfiguración en la propiedad y tenencia de la tierra. Si bien el tema es bastante amplio y estudiado desde diversas ópticas, el presente artículo de corte etnográfico busca abonar al tema desde la antropología política, a manera de entender cómo las relaciones de poder repercuten en los rasgos identitarios de algunas poblaciones con representación indígena a partir de un ejemplo de la especulación inmobiliaria en el estado de Yucatán, México.
\end{abstract}

Palabras clave. Estructura de poder, Antropología Política, Rasgos identitarios. X’men.

\begin{abstract}
Talking about the ejido in México refers us to a cluster of ideas associated with re and post revolutionary elements, that are largely related to the visibility of social actors without economic participation, lacking social development possibilities, as well as neoliberal reconfiguration maneuvers in property and tenuere. Although the subject is quite broad and studied from different perspectives, this paper rather than focusing on issues that properly refer to the change of economic model that went from a State characterized by promoting agricultural distribution, to a privatizing State, intends to pay the subject from cultural history and political anthropology, in order to understand how power relations have an impact on the identity features of some populations with indigenous representation.
\end{abstract}

Keywords: Power structure, Political Anthropology, Identity features, X’men.

\section{RESUMO}

Falar do ejido no México nos remete a um conjunto de ideias associadas a elementos re e pós-revolucionários, em grande parte relacionados à visibilidade de atores sociais com pouca ou nenhuma participação econômica, sem possibilidades de desenvolvimento social, bem como com manobras neoliberais. de reconfiguração da propriedade e da posse da terra. Embora o assunto seja bastante amplo e estudado sob várias perspectivas, este artigo etnográfico busca atentar para o assunto a partir da antropologia política, a fim de compreender como as relações de poder afetam os traços identitários de algumas populações com representação indígena a partir de um exemplo de imóveis. especulação no estado de Yucatán, México.

Palavras-chave: Estrutura de poder, Antropologia Política, Traços identitários. X Men.

\footnotetext{
${ }^{1}$ Fragmento de la entrevista realizada a Don Germando Euan, ejidatario de Sierra Papacal
} 


\section{INTRODUCCIÓN}

El presente trabajo tiene como objetivo el análisis de las estructuras de poder comunitarias asociadas a rituales y creencias relacionadas con saberes agrícolas y su afectación a partir de la venta de terrenos ejidales como consecuencia de la especulación inmobiliaria que desde inicios del siglo XXI se convirtió en una realidad cotidiana en el estado de Yucatán, México.

Se plantea cómo las relaciones de poder no son exclusivas del aparato político gubernamental, ni de grupos antagónicos que detentan cargos, ya sean gubernamentales o administrativo; o el control de recursos escasos. Sino que las relaciones de poder van más allá, y pueden ayudarnos a comprender desde otras ópticas, los cambios, o las pérdidas de rasgos culturales que otrora fueran elementos de la cotidianidad.

Lo anterior es relevante ya que una de las repercusiones, sobre todo en el ejido yucateco de la zona norte, fue la especulación inmobiliaria, con lo que diversos empresarios se apuntaron por la compra y venta de terrenos ejidales para la construcción de casas, plazas comerciales y fraccionamientos; y para el caso que nos compete, un Parque Científico y Tecnológico. Si bien el tema puede ser tratado desde diversos enfoques, nos toca orientar la problemática desde la historia cultural y de la antropología política, a través del análisis de las estructuras de poder que en algún momento sirvieron de eje rector dentro de un contexto agrícola. Y que, al desmoronar algunos elementos propios del contexto, llevó a la pérdida de un tipo de identidad construida.

La forma de presentar el documento se centra en primer lugar en el establecimeinto de las pautas teóricas propias de la antropologia política y el reconocimiento de las estructuras de poder. Seguido se muestra la fundamentación metodológica que se complementa con la caracterización de los actores sociales imersos en la problemática que se desarrolla. Asimismo se hace um breve recorrido etnográfico para referenciar la zona de estúdio. Posteriormente se presenta el apartado de dicusión em donde se aborda la estrutura de poder, seguido del impacto de la especulación inmobiliaria en la comunidade de estúdio.

\section{FUNDAMENTACIÓN TEÓRICA}

A continuación, se presenta un esbozo teórico en donde se desarrollan los conceptos relevantes para el texto, La manera en que se presenta este apartado es la siguiente: primeramente, se tratará de ubicar el trabajo dentro de la historia y la antropología sociocultural, haciendo énfasis en el reconocimiento de los inicios de ésta importante mancuerna. Asimismo, se abordarán los conceptos de poder y cultura, que nos brinden elementos para abonar a un análisis de la historia cultural de un ejido yucateco.

Sin embargo es pertimente señalar estudios recientes que abordan la problemática de la venta del ejido y el conflicto que genera en los distintos actores sociales que intervienen. Tal es el caso de (Cortez, 2018), quien estudia precisamente la venta de terrenos ejidales con fuentes hídricas naturales, como lo son, los cenotes. La autora refiere el conflicto que genera el modelo económico neoliberal, como una de las fuentes principales de la venta, por medio de la mercantilización de los recursos.

Otro estudio reciente en torno al ejido, es el que presenta (Barrera, 2019) al cuestiorse sobre la ingerencia de organismos internacionales en la política agrícola del ejido mexicano. Al respecto la autora señala que existe un impacto que muchas veces es poco visible pero que se concentra en dos ámbitos "visión empresarial vs visión cominidad: mientras que las directrices internacionales agrícolas de la OCDE y la FAO corresponden al sector empresarial, el sistema del trabajo del ejido corresponde a un sistema tradicional heredado desde la pre-colonia" (Barrera. 2019: 87). 
Asimismo, un estudio de (shumacher et. al, 2019), aborda el tema de la venta del ejido para favorecer el desarrollo urbano, desde el cuestionamiento sobre el papel que juegan las autoridades en la regulación y cambio en la tenencia de la tierra. Éste último trabajo sin duda comparte con el ensayo que se presenta acontinuación, el enfoque del desarrollo urbano, a partir de la posibilidad de la venta del ejido como conseciencia de la certeza jurídica que se produjo desde la economía neoliberal. Sin embargo la forma de entender nuestro problema de investigación, parte de comprender las dinámicas destro de una estructura de poder, para ello es necesario abordar algunos conceptos clave.

Uno de los conceptos de interés para el presente trabajo es el de antropología política. Al respecto Michel Smith (Michel Smith, autor del prólogo del libro Antropología Política (1979) de José Llobera) menciona que éstas se formaron bajo el estigma de la malversación del uso del conocimiento del otro orientándolo a procesos de colonización. Este campo de estudio es resultado de la conciliación entre la filosofía social y la ciencia política, para hacerse cargo de responder cuestionamientos de índole un poco alejada de la rigidez jurídica, ya que parte fundamental de su metodología radica en el soporte empírico, sin embargo, la antropología política se consolida hasta 1940, con la publicación de African Political System de Evans-Pritchard y Fortes.

Las primeras dos décadas luego de su consolidación su estudio se orientó hacia las tipologías y estructuras políticas. Luego de ello, Elizabeth Colson (Elizabeth Colson, autora del panorama teórico del libro Antropología Política (1979) de José Llobera) vislumbra un quiebre en los intereses de estudio, que canalizaron las investigaciones hacia el entendimiento de las desigualdades entre los actores sociales, dando pie al estudio sobre los conflictos. Si bien no se menciona como tal, en este sentido se podría ubicar una veta hacia los cuestionamientos de poder, desde la sociología del conflicto de Gluckman y Simmel. Si bien la sociología del conflicto de Gluckman en un inicio fue cuestionada por tratar al conflicto como la manifestación de intereses dentro de un sistema (rebelión), sin cuestionar al mismo sistema, la apertura a la visibilización de facciones dentro de un grupo pudo introducir nuevos focos de atención hacia los conflictos suscitados por la búsqueda del poder.

Por su parte George Balandier en Antropología política (2005) habla sobre la lucha que atravesó la antropología política para poder ser reconocida por disciplinas como la Historia. De modo que se propuso desde un enfoque que manejó la premisa de que la actividad política, no recae únicamente en las grandes instituciones o en los actores más relevantes de un periodo de estudio, sino que el estudio de lo político también abarca a los actores que no se encuentran dentro de lo institucional, dando de esta manera entrada al estudio de la política desde los subalternos.

De igual manera la antropología política abogó por la necesidad de ubicar el contexto cultural, ya que cada sistema político se encuentra inserto en uno, asumiendo así, las particularidades en cuanto a los conflictos que cada contexto pueda suscitar. En ese sentido, como menciona Balandier "Es pues, por efecto de una necesidad devenida manifiesta que la [...] la antropología, y la sociología política, así como la historia, han debido aunar sus esfuerzos. Y este encuentro otorga nuevo vigor a las previsiones de Durkheim. -Estamos convencidos... que un día vendrá en el que el espíritu histórico y el espíritu sociológico, no se diferencien más que por matices-" (Balandier, 2005: 90).

Como parte de este ejercicio conceptual, se debe apuntar también el concepto de política, ya que es bajo este contexto y sobre las decisiones hacia lo público que se desarrolla el tema del reparto agrario, y posteriormente la contrarreforma agraria. Aunque, como se mencionó en líneas anteriores la Antropología Política y la Historia Cultural sustentan que lo político no debe mirarse únicamente desde las instituciones o las grandes historias, sino que es necesario observar a los actores sociales que interactúan en otros contextos pero que son parte de la complejidad del tema político.

En este caso los ejidatarios, son quienes crean vínculos, asociaciones, relaciones y estructuras de poder. Si bien el concepto de política ha atravesado diversas críticas, en algunos casos por pretensiones universalistas y en otros casos por dejar fuera algunas tipologías, al respecto Ronald Cohen (1979) hace una exhaustiva revisión de los clásicos que comenzaron a esbozar una 
definición adecuada a política y lo político desde lo social, entre los que se encuentran Smith (1966), Easton (1965), Swart, Turner y Turden (1966), J. Levy (1966), Radcliffe-Brown (1940), Steward (1938), Fried (1964).

Para el presente trabajo se utiliza la definición de Swartz (1968), donde menciona que "se refiere a los (procesos) que están implicados en la determinación e implementación de objetivos públicos y/o en la distribución diferencial del poder y de su uso al interior del grupo o grupos involucrados en los objetivos que están siendo considerados"( La definición fue traducida al español por Roberto Valera (1984) en la página 19 del libro Expansión de sistemas y relaciones de poder), para nuestro caso, se puede observar a diferentes actores y grupos sociales inmersos en una dinámica que converge el ejercicio del poder, entre los que se encuentran los ejidatarios, los comisarios ejidales, los no ejidatarios, la población general sin derecho agrario, la autoridad gubernamental y la religiosa ${ }^{2}$, todos estos actores partícipes, de manera consiente o no, en objetivos públicos, bajo una distribución diferenciada del poder.

Es momento de adentrarnos en el análisis de la estructura de poder, para ello, valdría la pena definir por sí mismo el término poder, ya que, "El PODER es un tema común a todas las ciencias sociales [...] se ha utilizado con tal diversidad de significados que la búsqueda seria de sus variedades se convertiría pronto en una historia intelectual de una gran área del pensamiento social." (Adams, 1983:19)

Adams define al poder como "una relación social que descansa en algún patrón de controles y es reciproca. Es decir, ambos miembros de la relación actúan en términos de su propio interés, y específicamente lo hacen en términos de los controles que cada uno tenga sobre elementos que interesen al otro" (Adams, 1983: 39). Ahora bien, una estructura de poder va a ser definida por Adams como "cualquier conjunto sistémico de relaciones a través de las cuales los actores o las partes manifiestan sus preocupaciones relativas por el control sobre el ambiente y el poder sobre sus semejantes." (Adams, 1983: 37).

\section{PROCEDIMENTOS METODOLÓGICOS}

La unidad de análisis seleccionada para la realización del presente artículo de corte cualitativo, fue la comunidad de Sierra Papacal, comisaría de Mérida que se encuentra a $32 \mathrm{~km}$ del centro la ciudad, y a 23 kilómetros de la costa yucateca. Esto debido a que gran parte de sus terrenos ejidales fueron vendidos para la construcción del Parque Científico y Tecnológico de Yucatán (PCyTY). El cual tenía como principal objetivo, la construcción de un recinto que pudiera albergar distintos centros de investigación científica y académica. El recinto inició su edificación en 2008, durante el gobierno de Ivonne Ortega Pacheco y se intensificó durante la gestión de Rolando Zapata Bello.

La forma de abordar el tema fue a través del análisis de datos desde la antropología política, por medio del reconocimiento de las estructuras de poder de la comunidad gracias a una revisión etnográfica y entrevistas en profundidad bajo la técnica de bola de nieve. Así como de la historia cultural, en donde, a partir de la utilización de fuentes de Archivo, localización de fuentes hemerográficas, así como la utilización de algunas fuentes literarias y cinematográficas, fue posible conjuntar una visión histórico-antropológica que da cuenta de un tipo de estructura que permite comprender el cambio en algunos rasgos culturales identitarios de la comunidad de estudio.

\footnotetext{
${ }^{2}$ Como autoridad religiosa, se toma como referencia al $X^{\prime} M e n$, debido a que este personaje actúa en los rituales agrícolas como medio entre la población, la naturaleza y las creencias respecto al porvenir. De igual manera se reconoce en este trabajo como autoridad, debido a que es poseedor de conocimientos sobre rituales desconocidos por el grueso de la población.
} 
El informante principal de este trabajo es Don Germando Euan, ejidatario pensionado de 73 años y comisario ejidal en tres periodos consecutivos luego de 1984. Fue a partir de Don Germando que se pudo obtener contacto con el comisario ejidal, quien posteriormente me remitió con otros sujetos. Cabe señalar que el primer acercameinto con la problematíca radica en una estancia de seis meses en una unidad habitacional creada dentro del PCyTY, para albergar a investigadores y estudiates. El personal de limpieza y mantenimiento era por lo general, miembro de la comunidad de estudio, la cual previamente vendió parte de sus terrenos ejidales. De manera que, el primer acercameinto a la problemática que se presenta en el ensayo, fue a partir de las relaciones contruidas con ejidatarios o familiares directos que dejaron el trabajo agrícola para incorporarse al sector servicios dentro del PCyTY.

Las categorias utilizadas como forma de acercameinto a la realidad social fueron las siguientes: 1) Derechos de propiedad ejidal. 2) Vinculación con el trabajo agrícola. 3) Ritualización del ciclo agrícola. Dichas categorias de análisis contribuyeron al entendimento de la problemática al referir los aspectos principales para reconocer las estructuras de poder. De ahí tambien se desprende la importancia que el presente ensayo le otorga a la figura del $X^{\prime} m e n$.

En cuanto a las limitaciones durante el trabajo de campo, se puede referir la desconfianza de algunos ejidatarios de tocar el tema de la primera categoría de análisis (derechos de propiedad ejidal), ya que se trató diretamente el tema de la venta, lo que desprendió un conflicto entre grupos de ejidatarios que no coincidieron en su momento tanto en la venta, como com el precio.

\subsection{Caracterización de los actores sociales}

Como parte del análisis dentro de la estructura de poder, se trabaja con tres actores principales: el X’Men, los ejidatarios, y la población general.

El X'Men, quien en últimas fechas se equipara al yerbatero del pueblo, tiene desde la cosmovisión maya, otros elementos a destacar, ya que, el yerbatero o curandero es un personaje diferente, conocido como Ahdzac-yah, el cual se caracteriza por la sanación mediante el uso de remedios herbolarios. En el archivo hemerográfico vertical de la biblioteca yucatanense, Bayardi, Manuel ángel. (1986, 30 de enero) La medicina y el pueblo mágico de los mayas, p. 12 Novedades de Yucatán, publica un artículo donde se menciona una equiparación entre los X'Menes y los sacerdotes mayas. Tanto éstos, como los Ahdzac-yahnes derivan del primer médico, conocido como Zámna, (a quien también se le conoce como el sumo sacerdote y personaje más sabio de los itzaes) "No obstante los libros aseguran que el X'Men dedicado a la medicina espiritual era ayudado por los Akimes, Chilames y Chaakes". Si bien el artículo no menciona los libros a los que hace alusión, se puede interpretar que la nota se refiere al libro del Chilam balam de Chumayel, que es el compilado de libros que relatan sucesos históricos como la creación, el inframundo y el papel de los dioses. Además de vaticinios referentes a la visión cíclica del tiempo que poseían los mayas. En cuanto a los Akimes y los chaakes, se puede entender como la intervención divina del Dios Yum Kimil o Dios de la muerte y Chaak, Dios de la lluvia, este último sobre todo por la estrecha comunicación durante el ritual del Chaá chaak, el cual tiene como principio la petición de agua en forma de lluvia para cumplir con el ciclo agrícola

El $\mathrm{X}^{\prime}$ Men se caracteriza por el uso de ritos y oraciones. Ambos personajes son figuras representativas prehispánicas, que lograron trascender a través de los siglos por la necesidad su contexto. En el Archivo hemerográfico vertical de la biblioteca yucatanense. Yates Rafael (1988, 11 de diciembre) Historia de la medicina tradicional de Yucatán, p. 5 Novedades de Yucatán, se aprecia una fotografía de una figura de barro prehispánica, en el pie de la fotografía se puede leer "CINCO X'Menes o curanderos en torno a un cuerpo [...]" En la misma página se encuentra una segunda fotografía en la que se aprecia el busto de la figura prehispánica de un anciano, visto de 
perfil, ataviada con grandes ornamentas, y con el fenotipo características de los rostros prehispánicos. Al pie de la fotografía se puede leer "El X'Men o curandero que, llegado el caso, también la haría de Pul-Yah o brujo". Asimismo Bayardi, Manuel ángel. (1986, 30 de enero) En La medicina y el pueblo mágico de los mayas, p. 12 Novedades de Yucatán relaciona la posición de poder de los X'menes con su labor para la comunidad "Es entonces cuando las tradiciones nos dicen que los médicos tenían un carácter patriarcal y divino. Los mayas los reverenciaban casi como a un dios viviente"

Esta emblemática figura que fue referenciada por la prensa yucateca como símbolo de conexión entre un pasado, que busca unir la vena nacionalista con el origen prehispánico, sirve a nuestra investigación como agente clave en la reproducción de la identidad agrícola y como autoridad legitimada de la población. Bayardi, Manuel ángel. (1986, 30 de enero) abre su artículo periodístico justamente bajo una idea nacionalista cuando habla sobre "la búsqueda del alma nacional [...] buscar el pulso de la patria en todos los momentos [...] descubrir la misión del hombre mexicano en la tierra". El artículo es un ejemplo de lo que menciona López Caballero (2010) sobre nuestra relación con el pasado prehispánico "la idea de que los indígenas contemporáneos son la "herencia viva" de ese mundo antiguo destruido por la Conquista, y, sobre todo, la idea de que ese lejano pasado representa el patrimonio glorioso de todos los mexicanos, son, en realidad, innovaciones del siglo XX". En ese sentido se puede notar cómo la reivindicación de lo "maya" atiende a lo que José Luis Escalona (2017) menciona sobre la creación de una civilización milenaria, al vincular, con la población actual, los diversos estudios que se habían generado desde 1841, con Stephens y Catherwood; y los estudios de Evon Z. Vogt con el proyecto Harvard-Chiapas 1959 - 1984.

Los ejidatarios: La ficha técnica ejidal de inscripción para la certificación (30 de diciembre de 1998) estableció la cantidad de 287 ejidatarios registrados; 98 posesionarios y 28 avecindados. Si bien, el concepto de ejidatarios se ajusta a una política pública específica derivada del reparto agrario en favor de la población dedicada al cultivo, y que toma el nombre a raíz del término ejido. Al respecto, Roger Bartra (1978) quine hace un análisis de la población y situación agraria en México desde un enfoque marxista, por medio del estudio del ejido desde su estructura y las clases sociales, menciona que "es el producto de un proceso legal denominado dotación; las tierras las recibe un núcleo de población. En su origen, pues, no hay una compra: las tierras se obtienen gratuitamente, y proceden de haciendas expropiadas, tierras del Estado, etc. [...] El ejido es en principio, propiedad de la nación, pero cedida a una comunidad de campesinos en usufructo" (Bartra, 1978: 129 - 130). Esta política se intensificó durante la gestión del presidente Lázaro Cárdenas del Rio, con la creación de la Confederación Nacional Campesina, que tenía como objetivo la agrupación, bajo un mismo estandarte, de todos los actores sociales que tuvieran la intensión de trabajar el campo, por lo que en 1936 se funda el Departamento de Acción Social y Cultural y de Protección a los indios, desde una visión integracionista en donde se trataba de que, por lo menos desde lo legal, las categorías de indio, blanco y mestizo, no fueran determinante en el acceso a la tierra.

Para el caso de Yucatán, el ejido se dividió en dos zonas productoras, por una parte, la maicera y por otra la henequenera, esta última ubicada en la parte noroeste de la península, donde se encuentra actualmente nuestra comunidad de estudio. Lapointe y Dufresne, en su artículo, El cardenismo en Yucatán, mencionan que para 1935, la zona henequenera disponía de tres cuartas partes de una población que alcanzaba un total de 386,096 y más de la mitad de la tierra cultivada. De igual manera, la zona productora ha servido de inspiración para animar la fantasía y la cotidianidad de la que fuera llamada la época del oro verde. Uno de los momentos más representativos del siglo XIX y principios del siglo XX en Yucatán, fue sin duda el auge henequenero, que dio pie y origen a una cotidianidad particular, cargada de elementos reforzadores de poder y dominación desde un sector social hacia el resto de la población dedicada al campo. Esta relación trascendió los estudios formales de la historia y se observa en representaciones literarias, musicales y cinematográficas, una de las más representativas es la película La casta divina (1976) 
de Julián Pastor, en donde se expone la diferenciación entre peones y hacendados en Yucatán, bajo el contexto del auge henequenero, durante la intervención de Salvador Alvarado. Dada la importancia económica del Henequén, el desprendimiento de los hacendados de las tierras de cultivo fue complicado.

¿Qué no se han dado las dotaciones en Yucatán? ¿Por qué las tierras afectadas por la resolución presidencial no están cultivadas de henequén? Digo a ustedes, a nombre de la Revolución que las tierras deberían darse para que ustedes mismos sigan cultivando el henequén (Lapointe y Dufresne, (S/f): 349-350 citan discurso de Lázaro Cárdenas en Yucatán.)

Marie Lapointe y Lucie Dufresne, hacen un análisis de lo que representó el cardenismo para Yucatán, sobre todo en cuanto a la producción de henequén, para lo que mencionan que desde 1930 hubo una caída en las exportaciones, lo que fue incrementando año tras año, sin embargo se mantuvo un apoyo constante al campo bajo la idea de que por medio del ejido, los costos de producción podrían abaratarse, de manera que se autorizaron créditos con el objetivo de obtener beneficios a largo plazo. Cárdenas sin duda llegó a reconfigurar todo un sistema, y en cuanto a la imagen que representa, también fue blanco para la producción literaria, como lo es una novela biográfica llamada Tierra Roja (2016) de Ángel Palou García, donde, por medio de la reconstrucción de vida, nos hace comprender el papel del hombre que terminó con la larga lista de caudillos, para convertirse en el hombre de la institucionalidad. El porte y simbolismo de este personaje clave en la historia de México, fue también arrastrado al contexto cinematográfico desde un ambiente yucateco posrevolucionario hasta finales de los años treinta, con la película Un embrujo (1998) dirigida por Carlos Carrera, en donde Cárdenas se expone como el hombre encargado de establecer el orden social y en quien, el protagonista, deposita su fe para recuperar el trabajo del que fue injustamente despojado. La trama también nos lleva a una experiencia etnográfica de la vida cotidiana de un poblado yucateco, donde transcurre la vida de los protagonistas. Factores de lo cotidiano como la presencia del $\mathrm{X}^{\prime} \mathrm{Men}$, el contexto del reparto agrario, el sindicalismo y el mercado del henequén influyen en los actores que se desenvuelven en distintas categorías, como los indígenas, los mestizos y los migrantes.

Por su parte, Othón Baños menciona que Lázaro cárdenas tenía la firme idea de que los ex peones, los ex comuneros, y en general los personajes a cargo de la tierra, de manera organizada eran los destinados a transformar la vida posrevolucionaria, disminuyendo así, las desigualdades de la sociedad mexicana. De ahí que cita al general "Un ejido raquítico o miserable es la negación de la revolución mexicana" (Baños, (S/f): 401)

Para nuestro caso de estudio, el comisario ejidal comentó que la comunidad ejidataria derivó principalmente del agotamiento de la Hacienda que ellos denominan como Hóbonyá, la cual se encuentra a la mitad del camino entre Sierra Papacal y el puerto de Chuburná, hoy en día abandonada. Atendiendo a la memória de algunos pobladores, mencionan que sus abuelos, nacidos en promedio en la década de los veinte del siglo XX, nacieron en el poblado de Sierra Papacal. Mientras que los bisabuelos nacieron en las haciendas cercanas.

La población general: si bien, no se pudo localizar algún documento en el Archivo de la comisaría ejidal de Sierra Papacal que constara la fecha de fundación del poblado, la ficha técnica del Registro Agrario Nacional menciona que la primera dotación de tierra ejidal se realizó el siete de noviembre de 1924 (PHINA, núcleo agrario. Ficha técnica de Sierra Papacal Yucatán) otorgando el benefició a 160 personas. Lo que contrasta con la memoria colectiva de los ejidatarios que adjudican la primera dotación a Felipe Carrillo Puerto, quien concluyó su mandato en 1923, por lo que cabe la posibilidad que la gestión de las tierras fuera de este personaje y concluyera al año siguiente. Al respecto Othón Baños menciona que "La reforma agraria, impulsada por el gobernador Felipe Carrillo Puerto (1922-1923), entregó tierras incultas exclusivamente a los peones 
libres radicados en los pueblos, ya que La Ley de la Reforma Agraria vigente no preveía el caso de los acasillados que radicaban en las propias haciendas" (Baños, (s/f): 407)

Como se mencionó en líneas anteriores, las entrevistas realizadas arrojaron que la memoria los lleva a ubicar a la primera generación nacida en el pueblo a principios de los años 20, por lo que el poblado pudo derivar de las haciendas henequeneras cercanas.

En 1939, bajo el designio del presidente Lázaro Cárdenas hubo una expansión en la dotación de terrenos ejidales y otra ampliación hasta 1984. En 1992 con la contrarreforma agraria del presidente Carlos Salinas de Gortari, entró en vigor el Programa de Certificación de Derechos Ejidales y titulación de solares (PROCEDE), sin embargo, fue hasta 1998 que la certificación incidió en el ejido de Sierra Papacal.

Dentro del marco normativo, el programa hace referencia a la reforma al artículo $27^{\circ}$ de la Constitución mexicana y tiene como objetivo "dar certidumbre jurídica a la tenencia de la tierra a través de la entrega de certificados parcelarios y/o certificados de derecho de uso común, o ambos según sea el caso, así como de los títulos de solares en favor de los individuos con derechos que integran los núcleos agrarios que así lo aprueben o lo soliciten”. (Manual de PROCEDE, 2003:1).

\subsection{Un breve recorrido etnográfico}

La comunidad se caracterizó por su lejanía, hasta antes de la construcción del PCyTY debido a la falta de caminos que los comunicaran con los poblados cercanos. Las líneas ferroviarias al sur del poblado, eran las utilizadas para la transportación del henequén. La electricidad llegó durante la gobernatura de Carlos Loret de Mola Mediz, entre 1970 y 1976. Lo que generalizó prácticas propias de una comunidad aislada, como el cultivo de alimentos básicos para el autoconsumo como el maíz, el chile, la calabaza y el frijol; y la compra de hielo por veinte centavos a los vendedores ambulantes que llegaban de Mérida, el cual se envolvía en telas para su conservación, debido sobre todo al clima tropical propio de la selva baja yucateca.

La ingesta de carne, hasta antes de la llegada de la electricidad, también era deficiente, ya que, aunque tuvieran algún ejemplar de ganado mayor o un cerdo, este no podía ser consumido en su totalidad debido al reducido número de pobladores, teniendo como única solución el salado de la carne para su conservación. La comida principal para los ejidatarios durante su jornada, hasta la llegada de la electricidad fue el pozole, el cual preparaban con agua de los pozos de sus milpas y en ocasiones lo endulzaban con miel o lo sazonaban con chile.

La falta de luz limitaba las actividades cotidianas de la población hasta las siete y las ocho de la noche, para comenzar a las tres de la mañana. Había una clara división del trabajo que llevaba a la mujer a la preparación de los alimentos, el cuidado de los hijos pequeños y el cuidado de los animales de traspatio. Mientras que los hombres y los hijos en edad de trabajar regresaban de la milpa alrededor de las nueve de la mañana, para evitar la insolación.

Los niños comenzaban a seguir los pasos de sus padres alrededor de los 11 años, los acompañaban a la milpa y aprendían por imitación, el trabajo de la tierra. El dinero que ganaban por su trabajo era administrado por las mujeres, quienes se encargaban de comprarles ropa y huaraches de suela de llanta y ataduras de henequén. Una forma de diversión era la fiesta del santo patrono, San Antonio de Padua, cuya celebración se realiza hasta la actualidad en el mes de octubre. En la década de los 50, los bailes jaraneros en honor al santo se volvieron populares, y se convirtieron en la fecha más esperada de los niños y los jóvenes, quienes trabajaban en la milpa durante un año, para poder pagar los cinco pesos de las entradas. 


\section{Estructura de poder, el papel de $X^{\prime} M e n$ en la construcción de identidad}

Para el tema que nos compete, es necesario ubicar un elemento principal en la dinámica de poder, este es: la búsqueda del control de los recursos. Este control de los recursos es el ejercicio del poder que se manifiesta dentro de la estructura. El control sobre la tierra será entonces el objeto de deseo y por ende los actores sociales actuarán conforme a los intereses sobre este recurso.

Claramente podemos ubicar que un actor clave para ejercer control en este sistema es el comisario ejidal desde el ámbito institucional, ya que es el encargado muchas veces de informar y distribuir apoyos gubernamentales, así como es el primer contacto en los trámites legales para la venta de tierras ejidales, o para el ingreso de comuneros o avecindados que pretendan trabajar la tierra.

Pero recordemos que el objetivo de este ensayo es comprender más allá de la formalidad, por lo tanto, ubicamos que si bien, la tierra sí es objeto de deseo, para nuestros agentes (ejidatarios, población) el deseo no es tanto la tierra, sino lo que puede salir de ella, en otras palabras, lo que puede producir, ya que el producto de la tierra se convierte en el acceso a la subsistencia.

Es en este momento que ubicamos al $\mathrm{X}^{\prime}$ Men como el actor social clave dentro del ciclo agrícola, debido a que es el encargado de ritualizar la actividad, siendo el medio de comunicación entre la naturaleza, la divinidad y los hombres, en donde se manifiestan diferentes objetivos:

Primeramente, la petición a los señores del monte de poder cultivar en sus tierras, en esta actividad, el X'Men comunica por medio de un ritual a los "legítimos dueños de a tierra" la petición de los hombres de cultivarla. Ya durante el ciclo agrícola, este actor es el encargado de realizar el Cha'a chaak, que es la petición de lluvia para levantar la cosecha. En este punto se observa cómo la legitimad resulta en elemento clave que contribuye a la estructura de este sistema de relaciones de poder dentro de una comunidad agrícola con tradición maya. Para Adams, la autoridad es el elemento sustancial de alguien dotado de poder; y la legitimad es obtenida cuando los que pertenecen a algún grupo social consideran que algo es legal. "La legitimidad es una potencialidad cultural acerca de una autoridad, una ley, un acto, o lo que sea, para que se conforme con "los principios reconocidos o las reglas o normas aceptadas" Así pues, una cosa es legítima cuando la gente conviene en que es de algún modo correcto, apropiado o como debe de ser" (Adams, 1983: 48).

Una vez establecido que el $\mathrm{X}^{\prime}$ Men posee legitimante un tipo de poder sobre la sociedad por su capacidad de controlar los recursos espirituales dentro de un ciclo agrícola que rige el modo de subsistencia de una comunidad, es momento de establecer cuál es el tipo de ejercicio de poder que aplica dentro de sus relaciones de poder. Siguiendo a Adams y para lo que compete a la investigación, es necesario hacer notar que existen dos formas de poder, el poder independiente y el poder dependiente, la diferencia principal radica en que, en el primer caso, el poder es ejercido directamente por el actor que lo posee, y en el segundo, el actor transfiere su poder a otros pudiendo, ser por tres vías: concesión, delegación y asignación. Debido al tipo de conocimientos ancestrales del que es poseedor, el $\mathrm{X}^{\prime}$ Men ejerce un tipo de poder que no puede transferirse directamente para su ejercicio. Por lo tanto, su poder es independiente y legitimado social y culturalmente dada la necesidad de su contexto.

Pero ¿de qué manera se alcanza esta legitimidad traducida en la generación de identidades? Para responder a esta pregunta valdría la pena echar mano de la historia cultural, y de otros elementos en torno al poder. Primeramente, hay que aterrizar al ambiente como espacio de expresión y cultura. Al respecto Ricardo Pérez menciona que "la identificación de un espacio cultural no es solo producto del reconocimiento externo o interno; también depende de sus características particulares y de las interpretaciones que de ellas se han hecho y se hacen." Pérez Ricardo (2007) reflexiona sobre la formación de los estereotipos en los siglos XIX y XX, a partir de 
expresiones culturales que son interpretadas y difundidas, y que muchas veces se posicionan y arraigan como expresiones de identidad acorde a necesidades de carácter nacionalista.

La figura del $\mathrm{X}^{\prime} \mathrm{Men}$ en este caso, representa la expresión de conocimientos relacionados a saberes ancestrales que se manifiestan en la curación, sanación e invocación de deidades superiores, esto, por medio de la reproducción de la palabra y el movimiento del cuerpo, los cuales se transforman en mecanismos que reproducen y representan el simbolismo espiritual de las peticiones.

Sin embargo, aunado a este elemento, se debe tratar de entender ¿cómo una comunidad puede llegar a establecer, reproducir y difundir esta idea? Para esto ponemos atención en tres elementos: 1) La legitimación del $X^{\prime}$ men como poseedor de la sabiduría para pronunciar los rituales; 2) La aceptación de la dirección de la práctica ritual en el contexto que se establece y 3); El común acuerdo de los participantes sobre la veracidad de la celebración.

En nuestro caso, el $\mathrm{X}^{\prime}$ Men es el encargado de satisfacer la necesidad de una comunidad con una interiorización sobre la ritualidad en el campo, ya que funge como medio para reproducir de manera cabal los ciclos agrícolas. De modo que, para poder ejercer sobre la tierra, y aspirar al objeto de deseo entendido como la cosecha, es necesario el rito, el cual será ejecutado bajo los lineamientos establecidos a cargo de un actor legitimado, de manera repetitiva, arraigándose a la memoria, lo que pudo permitir su continuidad.

En este punto y como parte del sistema, se observa que el acto de la contemplación ritual del ciclo agrícola está cargado además de una compleja estructura funcional que contempla a diversos actores sociales, los cuales ejecutan acciones sistematizadas de forma colaborativa.

Las distintas actividades que se realizan durante la celebración de un rito agrícola, como lo es el caso del Chaá chaak son un ejemplo de la ejecución disciplinaria, lo que contribuye a su reproducción. En ese sentido Foucault menciona que "La disciplina procede ante todo a la distribución de los individuos en el espacio" (Foucault, 2003: 130). Sobre esto, el autor hace referencia al aparato disciplinario en torno a un espacio clausurado y de encierro. Para nuestra investigación el espacio clausurado lo establece el "monte" que, si bien es un espacio abierto, sin muros o barrotes, este resulta un espacio sagrado al que solo se puede acceder para convertirla en espacio cultivable, por medio de una petición, la cual se realiza por el X’Men durante un ritual. Cabe señalar que la petición para ingresar al monte no es exclusiva para el cultivo, en una de las entrevistas salió a relucir que, se debe mantener una buena relación con el señor del monte, pidiendo permiso para atravesarlo cuando se sale a cortar leña, o se tiene que atravesar el espacio para llegar a la milpa, de lo contario se corre el riesgo de perderse. Al respecto se señalaron los casos que en ocasiones se mencionan en algunos noticieros, en donde personas se pierden en el monte y aparecen algunos días después. Sobre eso mencionan que éstas no se pierden por el desconocimiento de los caminos, ya que han trabajado la misma tierra durante muchos años, más bien obedece a la intervención de los malos vientos que pueden rondar por el monte, de ahí la necesidad de tener una buena relación con las divinidades que habitan dicho espacio. Otro ejemplo de restricción del espacio se observa durante el ya mencionado ritual Chaá chaak, en el cual, se restringe o clausura la entrada a la milpa a las mujeres.

Otro de los elementos disciplinarios para Foucault se observa en la distribución de los individuos, en donde a cada uno de ellos le va a corresponder un lugar y una actividad precisa. Lo cual se observa también en el ejercicio de los rituales agrícolas, en donde si bien, la dirección está a cargo del $\mathrm{X}^{\prime} \mathrm{Men}$, existen actividades complementarias que están a cargo de otros sujetos, aunque actuando en conjunto como actores sociales.

Cabe señalar que, en este punto, una diferenciación entre los individuos recae sobre el ejidatario que haga la petición sobre su milpa, al cual se le atribuyen otras actividades como la de proveer alimento a todos los involucrados. Al respecto Foucault menciona que "en la diciplina, los elementos son intercambiables puesto que cada uno se define por el lugar que ocupa en una serie, y 
por la distancia que lo separa de los otros" (Foucault, 2003: 134), por lo que, lo importante en este caso, es el rango que se ocupa dentro de la dinámica, el cual, a excepción del $\mathrm{X}^{\prime} \mathrm{Men}$, es rotativo, dependiendo de la milpa en la que se haga la petición.

Aunado a la disposición disciplinaria necesaria para reproducir el ritual y que es determinante para el ciclo agrícola, podemos retomar el concepto de simulacro de Jean Baudrillard, en donde los actores sociales son participes de una teatralización simulada "Lo real es producido a partir de células miniaturizadas, de matrices y de memorias, de modelos de encargo y a partir de ahí puede ser reproducido un número indefinido de veces" (Baudrillard, 1978: 7) de manera que el ritual resulta la representación de una realidad otrora, pero que se adapta y se ejecuta como parte de una realidad que obedece a un contexto que continua dependiente de los elementos de la naturaleza y que se representan como divinidades a las cuales se le debe respeto por medio de la ritualización del medio.

\subsection{La venta del ejido y la pérdida de identidad}

¿Por qué hablar de una pérdida de identidad? Antes de la certificación, la comunidad se caracterizaba por una unidad espiritual en torno a los elementos que componían el ciclo agrícola, si bien, cada familia se organizaba para trabajar la tierra, acciones como la petición de permiso para cultivar y la petición de lluvias fungían como parte de un eslabonamiento que compenetraba a toda la población.

Al respecto los ejidatarios entrevistados recuerdan la manera en la que las personas se reunían para hacer los rituales para la petición de lluvia, en el cual se le otorgaba un carácter sagrado a la preparación de los alimentos, y se prohibía la participación de la mujer. Por su parte, los niños comenzaban a asistir a estas manifestaciones cuando el padre los empezaba a incluir en las labores de la siembra, alrededor de los 10 años.

La figura del X’Men era necesaria para la ejecución de los que llamaron rezos, los cuales realizaba el cerro yahuilu, que, según los informantes, se encuentra a dos kilómetros al norte. Se mencionó la gran precisión en las fechas de los rituales de Chaá Chaak, ya que una vez realizado del ritual, la lluvia caía en los dos días siguientes, lo que representaba la garantía para el sustento de las familias.

En la fototeca de la Biblioteca Yucatanense se pudo observar una colección fotográfica del antropólogo danés Christian Rasmussen (s/f) en donde se aprecian cuatro fotografías a color concernientes al ritual del Chaá chaak. En ellas se puede apreciar a un grupo de ejidatarios y un niño de aproximadamente ocho años preparando una ofrenda. Llama la atención que en una de las fotografías se observa una cruz verde ataviada con un hipil. Se preparan ofrendas de tipo tamal redondo con una marca de cruz hecha con semilla de calabaza molida. El sincretismo entre el simbolismo católico y el prehispánico es evidente, sobre todo cuando se sabe que originalmente la petición es directamente al dios de la lluvia Chaak pero en algunos casos, al preguntar sobre el ritual mencionan que la petición es a Dios, refiriéndose al Dios de los cristianos o al santo de nombre San Isidro, por relacionarlo con el agua. Si bien, la colección aparece en el archivo sin fecha, el antropólogo realizó un trabajo en Yucatán de nombre La milpa de los mayas en 1994, por lo que la colección puede ser parte de dicho proyecto

Sin embargo, este complejo entramado de símbolos y representaciones dejó de existir junto con el henequén, según los ejidatarios, hace alrededor de 30 años. Como nos cuentan, con la repartición de los terrenos y la certificación ejidal, llegó un desabasto en los apoyos para el campo, a demás de que la producción de la planta había perdido demanda, por lo que muchos ejidatarios vieron como una buena opción vender la tierra que, de cualquier forma, no podían trabajar, en palabras de ellos, el gobierno los dejó sin manera de poder defenderse. 
Ante este problema, los pobladores intentaron continuar con algunas costumbres, como la extracción de leña del monte para la venta, o la caza de venado, actividades que habían sido complementarias para el sustento familiar y que era parte de una cotidianidad que reflejaba la comunalidad de la tierra. Pero se encontraron con la privación al acceso a la travesía por los montes debido a que estos, ya tenían propietario. En este punto podemos observar cómo, el monte, que en algún punto fue un espacio clausurado y al cual solo se tenía acceso mediante la petición de permiso al señor del monte y a los aluxes, se clausura ahora bajo una normatividad institucionalizada. Se ve entonces como lo cotidiano pasó a convertirse en allanamiento y robo.

Esta problemática se hizo más evidente en el año 2005, cuando se vendieron los terrenos ejidales que después formarían parte del PCyTY, ya que, como parte de los contratos, se les hizo firmar un acuerdo que prohibía su tránsito por el monte. Las comunidades cercanas como Tamanché, Cosgaya, Xcanatun y Komchen se vieron en una situación similar cuando vendieron sus terrenos ejidales para hacer zonas residenciales de gama alta. Sobre esto mencionaron que, con la venta de los montes, se dio fin a los ejidatarios, refiriéndose a que dicho término solo se lleva de nombre, porque, al carecer de tierras, pierde el significado.

Con la pérdida del significado y la representación del concepto, también se perdió el elemento cultural que aportó a la memoria colectiva que se arraigaba por medio de los simulacros mencionados por Budriellard. Sin embargo, los datos parecen arrojar que el desuso del ritual, principalmente el Chaá chaak ocurrió algunos años antes de la parcelación y la venta de los ejidos, ya que el X’Men, piedra angular en la estructura de poder comunitaria falleció en 1984, sin que nadie que pudiera remplazar sus actividades, ya que como se mencionó, los X’Menes eran poseedores de conocimientos ancestrales y del vaticinio, al igual que tenían el control simbólico sobre los montes, al ser el vínculo entre la divinidad y los hombres.

Por códices traducidos y por relaciones franciscanas, según los enterados, el origen de la medicina de los mayas de Yucatán descansa sobre los sabios X'chel y el gran Zamná, en cuyo derredor surgen ídolos, figuras y nombres simbólicos que los sacerdotes y "X'Menes" se encargaban de difundir entre los núcleos esparcidos por la Península, para inculcar el fervor al más allá, al mismo tiempo que el temor de los castigos divinos que de hecho han controlado a enormes conglomerados de indígenas por siglos (Archivo hemerográfico vertical de la Biblioteca Yucatanense. Editorial (27 de agosto de 1989) Medicina y farmacopea prehispánica. Pp. 1-2. Suplemento cultural dominical año XXII, no. 992)

De manera que, cabría la interrogante de que si la pérdida de la figura del X’Men, algunos años antes de la parcelación y la venta de los terrenos ejidales contribuyó de alguna manera a que los pobladores accedieran de manera pronta a la venta de los montes. Si bien los entrevistados mencionaron que después de la certificación, la perdida de los apoyos y la baja demanda del henequén, la comunidad se encontraba económicamente en números rojos, no podemos dejar de lado la tradición, la cultura y las creencias que pervivieron a través de los años.

Además de esto, se debe señalar que las entrevistas arrojaron como punto de quiebre la desorganización, haciendo notar que la venta fue desigual por que no pudieron establecer un acuerdo que beneficiara a todos como grupo, de modo que cada uno vendió a diferentes precios. Con ello se puede interpretar que, dentro de la estructura de poder sobre el ciclo agrícola y la tierra, la pérdida del X’Men desmoronó la base de la colectividad, que se reforzaba con la ritualización del ciclo agrario, y tumbó a la única figura de autoridad legitimada, ya que el comisario ejidal, como parte de un acuerdo institucional, se observa más que como una autoridad, como un funcionario administrativo, susceptible a casos de corrupción.

\section{CONCLUSIÓN}


Ellos no van a poder trabajar la tierra, porque no hubo oportunidad de mostrarles cómo se hace

Lo anterior es un fragmento de la entrevista con Don Germando, refiriéndose a las nuevas generaciones, más específicamente a sus nietos, nos lleva a observar cómo los cambios en la política pública influyen en la cotidianidad y puede reconfigurar todo un sistema social. La pérdida de la tierra de cultivo por parte de los ejidatarios anuló la posibilidad de continuar con un modo de vida relacionado a la agricultura, sobre todo por que las tierras vendidas se utilizan como terreno para la construcción, por lo que, si bien, como mencionó don Germando, la tierra volvió a manos del patrón comparando a los compradores con los antiguos hacendados, los trabajadores del campo ya no podrán hacer uso de su conocimiento, ya que ese espacio, la milpa, pierde su significado y se transforma en terreno inmobiliarios. De manera que el patrón, será siempre quien tenga el poder para poseer la tierra.

De igual manera, a lo largo de este breve texto, se pudo observar la pertinencia de una mancuerna histórico-antropológica para adentrarnos en la realidad sociocultural de nuestra incumbencia. Se observó también cómo para nuestro caso, el desmoronamiento de un pilar ( $\left.X^{\prime} M e n\right)$ de la estructura de poder de la comunidad pudo influir en la pérdida de rasgos identitarios que posteriormente pudo allanar el camino para la venta de la tierra y del monte, que en algún momento tuvo el simbolismo poético de lo lejano y el miedo al futuro, lo que inspiraba sobre todo el respeto a lo divino, con relación a la cosecha.

Por lo tanto, la modificación al articulo $27^{\circ}$ de la constitución bajo los lineamientos de la política neoliberal en México de finales de los años 80, pudo, en menos de 30 años, desmoronar un sistema social y una estructura basada en la producción de la tierra, privando así, a las nuevas generaciones de los saberes propios de la agricultura y por el otro lado, sin proveer tampoco las herramientas para hacer algo más que un jardinero un parque científico.

Como se puede observar, el presente trabajo contribuye a las investigaciones relacionadas con el impacto de la aplicación de la política económica neoliberal en el sistema agrícola impulsado, sobre todo, luego de la Revolución mexicana, sin embargo, este breve ensayo es solo una mínima parte de todas las implicaciones que conlleva el ejido y su situación actual en México. Si bien en esta ocación nos encaminados a la estructura de poder y la ritualización, existen vetas importantes para darle una continuidad al problema de la especulación inmobiliaria en terrenos ejidales, tal es el caso de la polarización social respecto a la población y a los desarrollos de alta plusvalía en los que antes fueron terrenos de cultivo; o el conflicto de intereses y corrupción que surge del desacuerdo entre ejidatarios sobre la venta. Así como la relación de empresas inmobiliarias con personajes clave del gobierno y su influencia en el uso de la tierra.

\section{REFERENCIAS}

Adams, Richard (1983) Energía y Estructura. Una teoría del poder social. México: FCE

Balandier, George (2005) Antropología política. Buenos Aires: Ediciones del sol.

Baños, Othón (s/f) "Los nuevos campesinos de México" En Baños Othón (Ed) Sociedad, estructura agraria y Estado en Yucatán. Mérida: UADY

Bartra, Roger (1978) Estructura agraria y clases sociales en México. México: Serie popular Era, Instituto de Investigaciones Sociales/UNAM

Cohen, Ronald (1979) “El sistema político” En Llobera José (Coomp.) Antropología política. Barcelona: Anagrama. Pp. 27-53

Colson, Elizabeth (1979) “Antropología política” En Llobera José (Coomp.) Antropología política. Barcelona: Anagrama. Pp. 19-25

Cortéz, Inéz (2018) “Los cenotes en el mercado de tierras ejidales del oriente de Yucatán” En: revista Península vol.13 no.1 Mérida ene./jun. 2018.

Díaz, Rodrigo (s/f) "La celebración de la contingencia y la forma. Sobre la antropología de la performance”: Biblioteca jurídica virtual del Instituto de Investigaciones Jurídicas de la UNAM 
Escalona, José Luis (2017), "Encapuslated History: Evon Vogt and the Anthropological Making of the Maya". En López Caballero Paula y Acevedo Ariadna (eds.), Beyond Alterity. Producing Indigeneity in Modern Mexico. Arizona: Arizona University Press. Pp.244-262

Foucault, Michel (2003) vigilar y castigar. El nacimiento de la prisión. Buenos Aires: Siglo XXI Editores

Guardado, Gustavo y Torres Gabriela (2016) Antropología e Historia en México. Las fronteras construidas de un territorio compartido. México: El Colegio de Michoacán, CIESAS, CEPHCIS, UNAM

Gutelman, Michel (1980) Capitalismo y reforma agraria en México. México: Ediciones Era

Lapointe, Marie y Dufresne, Lucie (s/f) "El Cardenismo en Yucatán”. En Baños Othón (Ed) Sociedad, estructura agraria y Estado en Yucatán. Mérida: UADY

López, Paula (2010) “De cómo el pasado prehispánico se volvió el pasado de todos los mexicanos". En Fernando Escalante (coord.), La idea de nuestro patrimonio histórico y cultural. O de cómo hemos llegado a valorar y celebrar ciertas cosas nuestras. CONACULTA, México. Pp. 137-152.

Barrera, Telma (2019) "Las directrices internacionales agrícolas de la FAO y la OCDE y su impacto en el ejido en México". En: López, Teresa y Mártinez, (coord.) Reflexiones y discusiones de la administración pública en México. Versión electrónica: ISEC

Palou, Pedro (2016) Tierra Roja. La novela de Lázaro Cárdenas. México: Planeta

Pérez, Ricardo (2007) "El 'negro' y 'la negritud' en la formación del estereotipo jarocho durante los siglos XIX y XX”. En Expresiones populares y estereotipos culturales en México. Siglos XIX y XX. México: CIESAS. Pp. 175210.

Secretaría de la Reforma Agraria (2003) Programa de certificación de derechos ejidales y titulación de solares. México: INEGI RAN SRA

Schumacher, Melissa et. al. (2019) "Evolución y colapso de los ejidos en México: ¿Hasta dónde se utiliza la tierra comunal para el desarrollo urbano?" Preprintsdoi:10.20944/preprints201907.0302.v1

Smith, Michael (1979) "El estudio antropológico de la política" En Llobera José (Coomp.) Antropología política. Barcelona: Anagrama. Pp. 7-15

Varela, Roberto (1984) Expansión de sistemas y relaciones de poder. México: UAM

Páginas web

Registro Agrario Nacional

PHINA. Ficha Técnica del núcleo agrario de Sierra Papacal consultado julio de 2019 en: http://www.ran.gob.mx/ran/index.php/sistemas-de-consulta/phina

\section{Archivo}

Archivo vertical de la biblioteca yucatanense. Bayardi, Manuel Ángel. (1986, 30 de enero) La medicina y el pueblo mágico de los mayas. Novedades de Yucatán, p. 12

Archivo vertical de la biblioteca yucatanense. Yates Sosa, Rafael. (1988, 11 de diciembre) Historia de la medicina tradicional de Yucatán. Novedades de Yucatán, p.5

Archivo vertical de la biblioteca yucatanense Editorial. (1989, 27 de agosto) Medicina y farmacopea prehispánica. Novedades de Yucatán, p.1 y 2. Suplemento cultural dominical año XX11, no. 992

Archivo vertical de la biblioteca yucatanense. Rasmunssen, Christian. (s/f) Chaá Chaak. Colección Fotográfica. 